\title{
ALOCAÇÃO OTIMIZADA DE DISPOSITIVOS DE CONTROLE E PROTEÇÃO EM REDES DE DISTRIBUIÇÃO.
}

\author{
Luis Gustavo W. da Silva*† \\ lgwesz@gmail.com
}

\author{
Rodrigo A. F. Pereira ${ }^{\dagger}$ \\ ddigodyahoo.com
}

\author{
José R. Sanches Mantovani ${ }^{\dagger}$ \\ mant@dee.feis.unesp.br
}

\begin{abstract}
Optimized Allocation of Control and Protective Devices in Electric Distribution Systems

Optimized allocation and/or re-allocation of control and protective devices in distribution networks allows for a better operation and improvement of the reliability indices of the system. Control devices (used to reconfigure the feeders) are allocated in distribution networks to obtain an optimal operation strategy to facilitate power supply restoration in the case of a contingency. Protective devices (used to isolate faults) are allocated in distribution systems to improve the reliability and continuity of the power supply. It significantly reduces the impacts of faults in terms of customers' outages, and the time needed for fault location and system restoration. In this work, a binary non-linear programming model (BNLP) for the sectionalizing switches and protection devices allocation and/or re-allocation problem is presented. Constraints considered for the problem reflect technical and economical limitations, such as in-series protective devices coordination, number of available equipments, importance of the analyzed feeder, circuit topology, quality of power supply and physical limitations of the system. A dedicated Reactive Tabu Search Algorithm (RTS) is proposed for solving this problem. Results and optimized strategies for allocating or re-allocating protection devices and switches considering a real-life network restoration are presented.
\end{abstract}

KEYWORDS: Protection Systems, Restoration, Reliability, Reactive Tabu Search (RTS).

Artigo submetido em 28/05/2009 (Id.: 01008)

Revisado em 04/08/2009, 28/10/2009

Aceito sob recomendação do Editor Associado Prof. Eduardo N. Asada

\section{RESUMO}

A Alocação e/ou realocação otimizada de dispositivos de controle e proteção em redes de distribuição de energia elétrica melhora a qualidade do serviço de fornecimento de energia e os índices de confiabilidade do sistema. Neste trabalho apresenta-se um modelo de programação não linear binário (PNLB) para o problema de alocação e/ou realocação de chaves seccionadoras e dispositivos de proteção em alimentadores radiais aéreos de distribuição. As restrições consideradas no modelo refletem limitações técnicas e econômicas, tais como problemas de coordenação de dispositivos de proteção em série, número de equipamentos disponíveis, importância do alimentador sob análise, topologia do circuito, qualidade do fornecimento de energia e limitações físicas do sistema. Para solução desse problema propõe-se um algoritmo de Busca Tabu Reativo (RTS). São apresentados os resultados obtidos através de testes realizados com a implementação computacional da metodologia proposta, utilizando-se um alimentador de distribuição radial real com 134 barras.

PALAVRAS-CHAVE: Busca Tabu Reativa (BTR), Confiabilidade, Restauração, Sistema de Proteção.

\section{NOMENCLATURA}

$C C R, C C C$ e $C C I$ : Custos de energia das cargas residenciais, comerciais e industriais, não supridas por um determinado intervalo de tempo para que seja efetuados os remanejamentos das cargas para outra(s) seção(es);

$C F$ : Custo fi xo do equipamento de proteção e/ou controle; 
$\mathrm{CH}$ : Conjunto de pontos onde é permitido alocar chaves seccionadoras;

$C I_{i}$ : Custo anual total de interrupção para o alimentador $i$;

$C I P_{j}$ : Custo anual de interrupção devido a ocorrência de uma falta permanente na seção $j$;

$C I T_{j}$ : Custo anual de interrupção devido a ocorrência de uma falta temporária na seção $j$;

$C R R(),. C R C($.$) e C R I($.$) : Custo de energia das cargas$ residenciais, comerciais e industriais, não supridas por um determinado intervalo de tempo para que sejam realizado reparo na seção (.);

$C T R, C T C$ e $C T I$ : Custos de energia de cargas residenciais, comerciais e industriais, não supridas devido à incidência de uma falta temporária na seção $j$;

$d s_{j}$ : Variável que determina o tempo e a natureza da interrupção do fornecimento de energia da seção $j$, em função do tipo de dispositivo de controle e proteção que está alocado no inicio da seção $j, d s_{j}=1$ para religador, $d s_{j}=2$ fusível e $d s_{j}=3$ chave de manobras;

$F C C_{j}$ : Custo fi xo de instalação de chaves seccionadoras no ponto $j$

$F C P_{j k}$ : Custo fi xo de instalação de dispositivos de proteção do tipo $k$ no ponto $j$;

$G$ : $\quad$ Conjunto básico de laço em árvore para a topologia de um alimentador elétrico;

I: Taxa de atualização anual dos custos de interrupção de energia aos consumidores;

$j$ : Taxa de falta permanente média anual da seção $j$ ( falta $/ \mathrm{km} /$ ano $)$

$L: \quad$ Conjunto de seções a jusante da seção $j$;

$l_{j}$ : Comprimento da seção $j(\mathrm{~km})$.

$L R(),. L C($.$) e L I($.$) : Cargas residenciais, comerciais e$ industriais, conectada na seção (.);

$L T_{m}$ : Total de carga que deve ser transferida para os alimentadores vizinhos, a fi $\mathrm{m}$ de isolar a falta na secção $m$;

$M: \quad$ Conjunto de seções à montante da seção $j$ até a próxima seção com o $d s_{j}=1$ ou 2 ;

$M T$ : $\quad$ Conjunto de seções à montante da seção $j$, até a primeira seção com $d s_{j}=1$;

$N B_{i}$ : Conjunto de barras do alimentador $i$;

$N R_{i}$ : Número máximo de ramais do alimentador $i$;

$n \sec _{i}$ : Número total de seções do alimentador $i$. Defi nido em função da quantidade de dispositivos alocados;

$P_{D_{k}}$ : Demanda de potência ativa nodal da barra $k$;

$P j u_{j}$ : Custos de interrupção aos consumidores das seções à jusante da seção $j$ devido à faltas permanente na seção $j$
$P \mathrm{Po}_{j}$ : Custos de interrupção aos consumidores das seções à montante da seção $j$ devido à faltas permanente na seção $j$;

$P p o_{j}$ : Custos de interrupção aos consumidores devido à faltas permanente na seção $j$;

$Q: \quad$ Conjunto de pontos onde é permitido alocar dispositivos de proteção;

$Q_{D_{k}}: \quad$ Demanda de potência reativa nodal da barra $k$;

$R S$ : $\quad$ Capacidade de reserva dos alimentadores vizinhos de $i$ $\left(\Omega_{i}\right)$

$S_{k}$ : $\quad$ Potência ligada ao nó $k$ do alimentador $i$ e que deve ser remanejada para os alimentadores vizinhos;

$T$ : $\quad$ Horizonte de planejamento em anos;

$t_{0}$ : Tempo inicial do horizonte de planejamento;

$t_{k}$ : Tempo do estágio;

$T j u_{j}$ : Custos de interrupção de fornecimento de energia elétrica aos consumidores das seções à jusante da seção $j$ devido às faltas temporárias na seção $j$;

$T m o_{j}$ : Custos de interrupção de fornecimento de energia elétrica aos consumidores das seções à montante da seção $j$ devido à faltas temporária na seção $j$;

$T p o_{j}$ : Custos de interrupção de fornecimento de energia elétrica aos consumidores devido às faltas temporárias na seção $j$;

$V_{i}$ : Magnitude de tensão das barras dos alimentadores vizinhos de $i,\left(\Omega_{i}\right)$;

$V_{k}$ : Magnitude de tensão nodal da barra $k$;

$V_{k}^{\max }$ : Tensão máxima de operação permitida na barra $k$;

$V_{k}^{\text {min }}:$ Tensão mínima de operação permitida na barra $k$;

$X_{j 0}$ : Elo fusível alocado no ramal $j$ do alimentador $i$;

$X_{j i}$ : Religador alocado no ramal $j$ do alimentador $i$;

$X_{j k}$ : Variável binária de decisão $(0 / 1)$ de alocação de dispositivos de proteção do tipo $k$ no ponto $j$;

$Y_{j}$ : Variável binária de decisão $(0 / 1)$ de alocação de chaves seccionadoras no ramal $j$;

$\gamma_{j}$ : Taxa de faltas temporárias médias anuais da seção $j$ ( falta/km/ano);

$\lambda_{j}$ : Taxa de faltas permanentes médias anuais na seção $j$ ( falta/km/ano);

$\Omega_{i}$ : $\quad$ Conjunto de alimentadores vizinhos do alimentador $i$;

$\theta_{k}$ : $\quad$ Magnitude de ângulo nodal da barra $k$;

$\bar{X}_{i}$ : Número máximo de religadores que é permitido alocar no alimentador $i$;

$\bar{X}_{0}$ : Número máximo de elos fusíveis que é permitido alocar no alimentador $i$;

$\bar{X}_{0} s: \quad$ Número máximo de elos fusíveis que é permitido alocar em série no alimentador $i$;

$\bar{Y}: \quad$ Número máximo de chaves seccionadoras que é permitido alocar no alimentador $i$; 


\section{INTRODUÇÃO}

Curtos-circuitos, sobrecargas no sistema e falhas nos equipamentos são condições anormais de operação que estão sujeitos os circuitos de distribuição. Descargas atmosféricas, galhos de árvores que tocam os condutores, falhas de isoladores e interferências no sistema, tanto humanas como de animais, são geralmente os principais causadores da atuação dos dispositivos de proteção interrompendo o fornecimento de energia para os consumidores. Sob condições de faltas permanentes, o sistema passa do estado normal de funcionamento para o estado restaurativo conforme ilustrado na Figura 1 (Anderson, 1999). Os estados operacionais de um sistema podem ser definidos como: 1) Normal, quando as demandas de cargas e as restrições operativas do sistema estão sendo satisfeitas; 2) Emergência, quando as restrições operativas não estão sendo satisfeitas, e; 3) Restaurativo, quando houver uma interrupção parcial ou total do fonercimento de energia. Os índices de confiabilidade do sistema estão relacionados com o tempo de operação da rede em cada um desses estados previamente definidos.

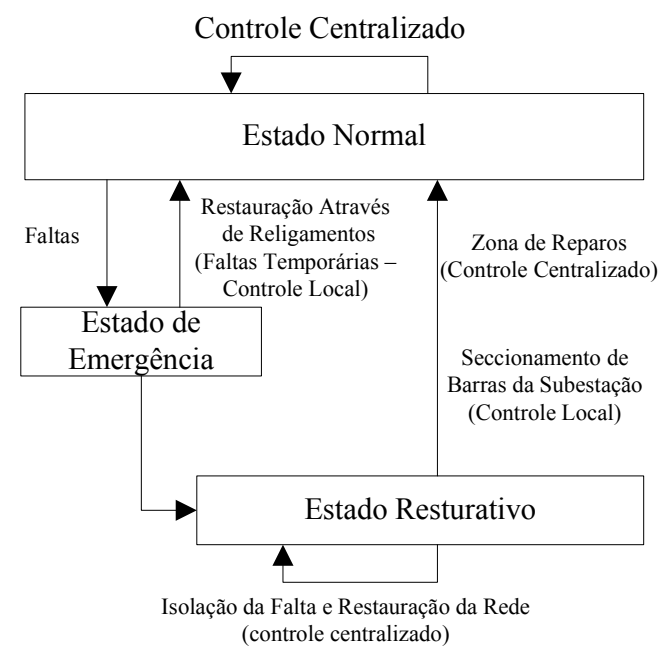

Figura 1: Estados de operação de um sistema de distribuição (Adaptado de Anderson(1999))

O planejamento ideal dos sistemas de distribuição, de forma geral, deve contemplar tanto a expansão da rede como a confiabilidade da mesma, mas sob o aspecto prático está limitado apenas à alocação de novas subestações e de novos alimentadores primários (Brown et al., 1996; Billinton and Jonnavithula, 1996). Após a alocação das subestações e dos alimentadores primários definindo a topologia da rede, o sistema de distribuição necessita ser projetado com mais detalhes e os aspectos relacionados com a confiabilidade da rede tornam-se mais relevantes. Uma das alternativas de melhoria da confiabilidade do sistema é através da alocação de chaves seccionadoras e dispositivos de proteção em pontos estratégicos da rede, visando a melhoria da qualidade do fornecimento e dos índices de confiabilidade.

Dispositivos de proteção alocados nos alimentadores primários são destinados a proteger o sistema elétrico contra faltas permanentes ou temporárias. Curto-circuitos, sobrecargas no sistema e falhas nos equipamentos, são condições anormais comuns em circuitos de distribuição.

Chaves seccionalizadoras permitem controlar a interrupção do fornecimento de energia devido às faltas permanentes e também para a execução de obras de expansão, e intervenções para manutenção preventiva em componentes da rede. Desta forma, a rede deve possuir um conjunto de chaves seccionalizadoras (automáticas ou manuais) para reconfiguração, interrompendo o fornecimento de energia para a menor quantidade de consumidores possível e remanejando alguns consumidores para os alimentadores vizinhos.

Alternativas que minimizam o número de dispositivos a serem manobrados devem ser adotadas, uma vez que, quanto maior o número de manobras, mais difícil é obter a coordenação e seletividade para o sistema de proteção e maior é o tempo gasto para estabelecer um plano de restauração da rede. Por consequência, maior o tempo de interrupção para os clientes. Em geral, o impacto causado aos consumidores devido a alocação de dispositivos de controle e proteção no sistema está relacionado com a frequência e duração da interrupção. Na literatura, diversos trabalhos tratam separadamente da alocação otimizada de chaves para restauração da rede (Billinton and Jonnavithula, 1996; Celli and Pilo, 1999; Levitin et al., 1994; Teng and Liu, 2003; Silva et al., 2004) e alocação otimizada de dispositivos de proteção (Soudi and Tomsovic, 1999; Soudi and Tomsovic, 2001; Silva et al., 2004).

Neste trabalho apresenta-se uma nova metodologia para efetuar a alocação otimizada de chaves seccionalizadoras e dispositivos de proteção, simultaneamente, no mesmo processo de otimização. No desenvolvimento do modelo matemático é considerada a possibilidade de restauração da rede de distribuição através da alocação otimizada de chaves seccionalizadoras quando ocorrem faltas permanentes em cada uma das k-ésimas seções dos n-ésimos alimentadores. Nesta modelagem consideram-se que os dispositivos de proteção operam de forma seletiva e coordenada, reduzindo a atuação imprópria do fusível com relação às faltas temporárias, através da atuação dos religadores. Outro aspecto prático e importante abordado nesta modelagem é considerar a alocação simultânea dos dispositivos de controle e proteção. Quando ocorre uma contingência, os dispositivos de proteção devem atuar a fim de proteger o sistema e 
os dispositivos de controle devem ser chaveados (abrir ou fechar) para isolar a área sob faltas, bem como transferir as cargas interrompidas para os alimentadores vizinhos. Os limites operacionais tais como, a capacidade de fornecimento das subestações e alimentadores vizinhos e a queda de tensão nos alimentadores entre outros, devem ser sempre respeitados.

Um algoritmo de Busca Tabu Reativo - BTR (Reactive Tabu Search - RTS) dedicado é proposto para resolver o modelo de otimização. Para ilustrar a aplicação desta técnica é usado um alimentador real, 13,8 kV, 134-barras, aéreo, trifásico e com o transformador da subestação em conexão triângulo-estrela aterrada.

\section{MODELO MATEMÁTICO}

O objetivo deste trabalho é o desenvolvimento de uma metodologia para alocação otimizada de dispositivos de controle e proteção em redes de distribuição, visando a melhoria dos índices de confiabilidade e qualidade de fornecimento de energia para os consumidores. $\mathrm{O}$ modelo matemático é desenvolvido considerando-se que o planejamento da expansão da rede já esteja concluído com a alocação das subestações, dos alimentadores primários, bem como dos ramais de interligação entre os alimentadores destinados à reconfiguração da rede. A topologia da rede após esta etapa de planejamento está ilustrada na Figura 2.

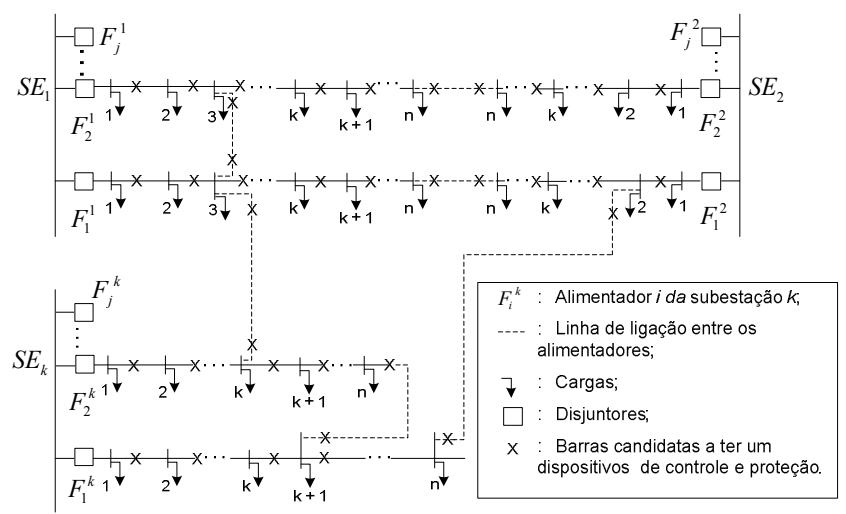

Figura 2: Sistema de distribuição após a fase de planejamento da expansão.

A alocação e/ou realocação otimizada de dispositivos de controle e proteção em redes de distribuição é a busca dos melhores locais para se alocar os diferentes tipos de dispositivos, possibilitando minimizar a soma dos custos de investimento na rede para reduzir as interrupções e os custos destas interrupções para os consumidores. O modelo matemático deve contemplar a obtenção de soluções ótimas técnicas e economicamente viáveis. Por exemplo, fusíveis são dispositivos de baixo custo, mas podem ser acionados tanto para faltas permanentes como temporárias. Uma vez que o número de faltas temporárias é grande, o uso de religadores pode ser econômico e tecnicamente mais adequado a longo prazo para proteger o sistema contra faltas temporárias (Anderson, 1999). Desta forma, no caso de faltas permanentes seria interessante a instalação apenas de fusíveis. Entretanto a porcentagem de ocorrência deste tipo de falta é muito pequena em relação à porcentagem de ocorrência de faltas temporárias, de modo que o modelo matemático deve contemplar tanto a alocação de religadores como de fusíveis, deixando para os religadores o papel de eliminar as faltas temporárias e de proteção de retaguarda para os fusíveis no caso de faltas permanentes, e para os fusíveis eliminar as faltas permanentes na região da rede de distribuição, onde os mesmos são proteção principal. Desta forma, o modelo matemático para alocação e/ou realocação de dispositivos de controle e proteção para cada alimentador i de uma determinada rede de distribuição pode ser matematicamente formulado como:

$$
\begin{aligned}
& \text { Min } C I_{i}=\sum_{j \in Q} F C P_{j k} X_{j k}+\sum_{j \in C H} F C C_{j} Y_{j}+ \\
& \sum_{k=1}^{T}\left(\sum_{j=1}^{n \text { sec }_{i}}\left(C I P_{j}+C I T_{j}\right)\right) \frac{1}{(1+I)^{t_{k}-t_{0}}} \\
& \text { s.a. } \\
& g\left(P_{D_{k}}, Q_{D_{k}}, V_{k}, \theta_{k}\right)=0 \quad k=1, \cdots, N B_{i} \\
& V_{k}^{\min } \leq V_{k} \leq V_{k}^{\max } \quad k=1, \cdots, N B_{i} \\
& \sum_{k \in i} S_{k} \leq R S j \in \Omega_{i} \\
& \sum_{j=1}^{N R_{i}} Y_{j} \leq \bar{Y} \\
& \sum_{j=1}^{N R_{i}} X_{j 1} \leq \bar{X}_{1} \\
& \sum_{j=1}^{N R_{i}} X_{j 0} \leq \bar{X}_{0} \\
& \sum_{j \in G} X_{j 0} \leq \bar{X}_{0 s}
\end{aligned}
$$

As restrições do problema de alocação otimizada de dispositivos de proteção são de natureza técnica e econômica. As restrições de natureza técnica estão relacionadas com a coordenação dos dispositivos de proteção e a topologia do sistema (número de dispositivos em série). As restrições de natureza econômica estão relacionadas com os custos de instalação e operação dos dispositivos, natureza e importância da carga. A equação (2) representa as equações de fluxo de potência para cada ramo e nó da rede de distribuição de energia elétrica (Leis de Kirchhoff). Equação (3) assegura que a magnitude de tensão para cada nó do 
circuito é mantida dentro de limites pré-estabelecidos. A equação (4) assegura que a capacidade de transferência de cargas do alimentador sob análise não ultrapasse a capacidade de transferência dos alimentadores vizinhos. As equações (5)-(7) estabelecem, respectivamente o número máximo de chaves seccionadoras, religadores e elos fusíveis que podem ser alocados no alimentador. A equação (8) estabelece o número máximo de fusíveis que pode ser alocado em série para obter coordenação entre os elos fusíveis, religadores e relés de sobrecorrentes. As restrições para o problema tais como: as limitações da capacidade do alimentador, Lei de Kirchhoff para corrente, fluxo de potência para as linhas, perfil de tensão, são analisadas e consideradas usando um algoritmo de fluxo de potência monofásico rápido e eficiente para redes de distribuição (Baran and $\mathrm{Wu}, 1989$ ). Em seguida discute-se detalhadamente a concepção filosófica do modelo matemático proposto para a função objetivo. $\mathrm{O}$ modelo representado pelas equações (1)-(8) é um problema de programação não linear com variáveis binárias relacionadas com a decisão de alocar dispositivos de controle e proteção em um conjunto pré-estabelecido de ramos candidatos.

\subsection{Modelo de Função Objetivo}

A função objetivo proposta (1) é a soma dos custos fixos devido à alocação e/ou realocação de dispositivos de controle e proteção, bem como os custos de interrupções para os consumidores calculados dentro do horizonte de planejamento e atualizados para o valor presente. Os custos médios das interrupções (tanto temporárias e permanentes) são usados para calcular o custo total de interrupções para os consumidores (Chowdhury and Koval, 1998). Estes custos podem ser avaliados a partir da análise do faturamento das empresas para o alimentador sob análise, ou simplesmente basear-se em um conjunto de padrões pré-estabelecidos pelas agências reguladoras, com suas penalizações associadas. Esses padrões geralmente medem o número e a duração das interrupções sentidas pelos consumidores. O System Average Interruption Frequency Index (SAIFI), bem como o System Average Interruption Duration Index (SAIDI), são exemplos adotados internacionalmente para tais índices de padrões (Soudi and Tomsovic, 1999; Soudi and Tomsovic, 2001).

O impacto que o processo de alocação de dispositivos tem sobre os custos de interrupção está relacionado com a variação da frequência e duração das interrupções sentida pelos consumidores. $\mathrm{O}$ custo de cada interrupção é calculado levando em consideração a localização de todos os dispositivos de controle e proteção no alimentador de distribuição radial. O alimentador é dividido em seções, de acordo com a localização dos dispositivos instalados, ou seja, cada dispositivo instalado separa duas seções: seção a montante do dispositivo, e a seção a jusante. Uma falta em qualquer seção irá afetar o restante das seções de maneira distinta, dependendo do tipo de dispositivos instalados. Portanto, os custos de uma interrupção dependem dos tipos de dispositivos instalados na região de influência da falta, isto porque o plano de restauração das cargas devido à incidência de faltas permanentes em cada seção depende destes dispositivos.

As seções da rede de distribuição são classificadas de acordo com o dispositivo instalado no seu início:

- Seções com religadores alocados no início $\left(d s_{j}=\right.$ 1): Os religadores possuem as funções de manobras e proteção para faltas permanentes e temporárias. $\mathrm{Na}$ incidência de faltas permanentes na seção iniciada por um religador, a falta é isolada apenas nesta seção, os consumidores das seções a jusante da seção sob falta podem ser restaurados (fornecimento através dos alimentadores vizinhos) e os consumidores das seções a montante do religador não sofrem interrupção de energia elétrica. Os custos de interrupção são os custos de reparo e de chaveamentos (remanejamento de cargas para alimentadores vizinhos). Na incidência de faltas temporárias na seção iniciada por um religador todos os consumidores a jusante do religador sentirão um desligamento transitório. O custo de interrupção nestas seções é um custo transitório de interrupção e os consumidores das seções a montante não sofrem interrupção do serviço de energia elétrica.

- Seções com chaves fusíveis alocados no início $\left(d s_{j}=2\right)$ : As chaves com elos fusíveis no modelo desenvolvido são consideradas que possuem apenas a função de proteção, e não apresentam a capacidade de chaveamentos. Para isolar as faltas que ocorrem neste tipo de seção, duas chaves seccionadoras, uma alocada à montante e outra a jusante do elo fusível, devem ser abertas. Os consumidores das seções adjacentes a estes dispositivos de manobras podem ser restaurados, ou seja, o fornecimento de energia para os mesmos pode ser realizado através dos alimentadores vizinhos desde que obedecida a restrição de mínima magnitude de tensão de restauração para rede. Os custos de interrupção são os custos de reparo e de chaveamentos (remanejamento de cargas). Na incidência de faltas temporárias na seção iniciada por uma chave fusível ocorre a atuação do dispositivo de proteção com religamento automático a montante mais próximo e em série com o elo fusível. Todos os consumidores a jusante do dispositivo de religamento automático sentirão o desligamento transitório. $\mathrm{O}$ custo de interrupção por estas seções será um custo transitório de interrupção. Os consumidores das seções à montante 
do dispositivo de religamento automático não sofrerão interrupção do fornecimento de energia elétrica.

- Seções com chaves seccionadoras alocadas no início $\left(d s_{j}=3\right)$ : As chaves seccionadoras não possuem a função de proteção. As chaves seccionadoras (manuais ou automáticas) e os religadores são considerados equipamentos para efetuar chaveamentos para isolar seções da rede sob faltas permanentes e remanejar cargas para os alimentadores vizinhos. Na incidência de faltas permanentes ou temporárias na seção iniciada por uma chave seccionadora, ocorre a atuação de um religador ou de um elo fusível a montante mais próximo e em série com esta chave seccionadora. Se o dispositivo de proteção sensibilizado é um fusível, a seção definida pela chave de seccionadora e a seção definida pelo fusível são isoladas. Caso o dispositivo de proteção que atuou seja um religador apenas, a seção definida pela chave seccionadora é isolada, os consumidores das seções a jusante podem ser restaurados (chaveamento para os alimentadores vizinhos). Os custos de interrupção são os custos de reparo e de chaveamentos (remanejamento de cargas). Na incidência de faltas temporárias na seção iniciada por uma chave seccionadora ocorre a atuação do dispositivo de proteção com religamento automático à montante mais próximo e em série com a chave seccionadoras. Todos os consumidores a jusante do dispositivo de religamento automático sentirão o desligamento transitório. O custo de interrupção para estas seções é um custo transitório. Os consumidores das seções à montante do dispositivo de religamento automático não sofrerão interrupção de energia elétrica.

\subsection{Custos de Interrupção}

Custo de interrupção devido à incidência de faltas permanentes na seção $j$ do alimentador $i\left(C I P_{j}\right)$, é dado por:

$$
C I P_{j}=\lambda_{j} l_{j}\left(P p o_{j}+P m o_{j}+P j u_{j}\right)
$$

Desta forma, os termos $P p o_{j}, P m o_{j}$ e $P j u_{j}$ são calculadas através das seguintes equações:

$$
\begin{gathered}
P_{P o_{j}}=\left(L R_{j} C R R+L C_{j} C R C+L I_{j} C R I\right) \\
P m o_{j}=\left\{\begin{array}{c}
0 \\
\sum_{m=1}^{M}\left(\begin{array}{l}
L R_{m} C C R+ \\
L C_{m} C C C+ \\
L I_{m} C C I
\end{array}\right), \text { Se } \mathrm{ds}_{\mathrm{j}} \neq 3 \\
\end{array}\right\}
\end{gathered}
$$

$$
P j u_{j}=\left\{\begin{array}{c}
\sum_{m=j+1}^{L}\left(\begin{array}{c}
L R_{m} C R R+ \\
L C_{m} C R C+ \\
L I_{m} C R I
\end{array}\right), \text { Se } d s_{j}=2 \\
\sum_{m=j+1}^{L}\left(\begin{array}{l}
L R_{m} C C R+ \\
L C_{m} C C C+ \\
L I_{m} C C I
\end{array}\right), \operatorname{Se}\left\{\begin{array}{l}
R S \geq L T_{m} \\
R S \in \Omega_{i} \\
d s_{j} \neq 2 \\
V_{i}>V_{\min } \\
V \in \Omega_{i i}
\end{array}\right. \\
\sum_{m=j+1}^{L}\left(\begin{array}{l}
L R_{m} C R R+ \\
L C_{m} C R C+ \\
L I_{m} C R I
\end{array}\right), \operatorname{Se} \begin{cases}R S<L T_{m} \\
R S \in \Omega_{i} \\
d s_{j} \neq 2\end{cases}
\end{array}\right.
$$

As parcelas que compõem o $C I P$ para qualquer seção $j$ do alimentador genérico podem ser interpretadas como:

$P p o_{j}$ : Para faltas permanentes na seção $j$, as cargas desta seção devem ser isoladas do sistema para que sejam efetuados os serviços de reparos. O custo é então associado ao tempo de interrupção de reparo. $P \mathrm{Po}_{j}$ : Para faltas permanentes na seção $j$, o tipo de dispositivo que define a seção $j$ tem que ser verificado para determinar os custos de interrupção:

- Para seções definidas por religadores automáticos $\left(d s_{j}=1\right)$ ou por elos fusíveis $\left(d s_{j}=2\right)$, as cargas a montante da seção não sofrem interrupções de fornecimento, pois tanto os religadores como os elos fusíveis atuam para faltas permanentes, ou seja, apenas as cargas das seções à jusante sofrem interrupção, e o valor de $\mathrm{Pmo}_{j}$ é igual a zero.

- Seções definidas por chaves seccionadoras $\left(d s_{j}=\right.$ 3): Algum dispositivo (religador ou elo fusível) a montante da seção atua, porém as cargas a montante das seções podem ser supridas normalmente, necessitando apenas de uma pequena interrupção para que possam ser realizados os chaveamentos necessários para a restauração do serviço. O custo é então associado ao tempo de interrupção para o remanejamento de cargas entre alimentadores.

$P j u_{j}$ : Para faltas permanentes na seção $j$, o tipo de dispositivo que define a seção $j$ tem que ser verificado para determinar o custo de interrupção:

- Elos fusíveis $\left(d s_{j}=2\right)$ : O tempo de interrupção das cargas a jusante desta seção é maior para que seja realizado o reparo da seção sob falta. Neste modelo, não está sendo considerada a possibilidade de ter uma chave fusível com capacidade de realizar manobras. O custo é então, associado ao tempo de interrupção para reparos na rede;

- Religador $\left(d s_{j}=1\right)$ ou chave seccionadora $\left(d s_{j}=\right.$ 3): As cargas a jusante desta seção podem ser 
remanejadas para um dos alimentadores vizinhos, caso haja capacidade de reserva suficiente nestes alimentadores e não haja problemas de violação na magnitude mínima das tensões de operação nas barras do sistema. Neste caso ocorre uma interrupção de curta duração para o remanejamento (chaveamento) das cargas desta seção para os alimentadores vizinhos. O custo é então, associado ao tempo de interrupção de chaveamento. Não sendo possível o remanejamento de cargas, haverá a necessidade também de uma interrupção de maior duração nesta seção até que seja executado o serviço de reparo na seção $j$. O custo é então, associado ao tempo de interrupção para reparo.

Os custos de interrupção devido à ocorrência de faltas temporárias no alimentador também são considerados. Os custos refletem os efeitos de uma interrupção de energia elétrica de curta duração devido à atuação de dispositivos de proteção com religamento automático. Estes custos são incorporados no modelo com o objetivo de minimizar os inconvenientes das faltas temporárias através da alocação adequada destes dispositivos. Os custos de interrupção devido à ocorrência de uma falta temporária na seção $j$ $\left(C I T_{j}\right)$, do alimentador $i$, são dados por:

$$
C I T_{j}=\gamma_{j} l_{j}\left(T p o_{j}+T m o_{j}+T j u_{j}\right)
$$

Os termos $T p o_{j}, T m o_{j}$ e $T j u_{j}$ são dados pelas seguintes equações:

$$
\begin{aligned}
& T p o_{j}=\left(L R_{j} C T R+L C_{j} C T C+L I_{j} C T I\right) \\
& T m o_{j}=\left\{\begin{array}{c}
0 \quad, S e d s_{j}=1 \\
\sum_{m=1}^{M T}\left(\begin{array}{c}
L R_{m} C T R+ \\
L C_{m} C T C+ \\
L I_{m} C T I
\end{array}\right), \text { Se } d s_{j} \neq 1
\end{array}\right. \\
& T j u_{j}=\left\{\begin{array}{c}
\sum_{m=j+1}^{L}\left(L R_{m} C T R+L C_{m} C T C+L I_{m} C T I\right)
\end{array}\right.
\end{aligned}
$$

As parcelas que compõem o CIT para qualquer seção $j$ de um alimentador genérico podem ser interpretadas como:

$\mathrm{Tpo}_{j}$ Para faltas temporárias na seção $j$, as cargas desta seção e à sua montante são desligadas temporariamente devido à incidência desta falta, através da atuação do religador, caso este esteja alocado nesta seção ou em alguma seção a sua montante, ou através da atuação do disjuntor equipado com relé na subestação. $\mathrm{O}$ custo é então, associado à interrupção de curta duração;

$T m_{j}$ : Para faltas temporárias na seção $j$, primeiramente verifica-se o $d s_{j}$ da seção:

- Religador automático $\left(d s_{j}=1\right)$ : as cargas a montante da seção $j$ não sofrem alterações no seu funcionamento, pois o religador atua para faltas temporárias, ou seja, apenas as cargas das seções à jusante são sensibilizadas pela interrupção, e neste caso o valor de $T m o_{j}$ é igual a zero.

- Chave de manobra $\left(d s_{j}=3\right)$ ou um elo fusível $\left(d s_{j}=2\right)$ : os dispositivos de proteção com religamento automático à montante desta seção e em série com o ponto sob falta devem atuar. O custo é então, associado à interrupção de curta duração.

$T j u_{j}$ : Para faltas temporárias na seção $j$, , todas as seções à jusante são sensibilizadas por esta interrupção devido a atuação de um religador na seção $j$ ou em alguma seção à montante de $j$, ou através da atuação do disjuntor equipado com relé na subestação. $\mathrm{O}$ custo é então, associado à interrupção de curta duração.

\section{TÉCNICA DE SOLUÇÃO}

Nesta seção apresenta-se a técnica desenvolvida para resolver o modelo de otimização proposto.

\subsection{Seleção dos Pontos Candidatos para Alocação dos Dispositivos de Controle e Proteção}

As regras heurísticas de seleção do conjunto de pontos candidatos à alocação dos dispositivos de controle e proteção são construídas baseadas na experiência dos engenheiros e técnicos das áreas de operação e planejamento das empresas. Esta heurística é composta dos seguintes passos:

- Alocação de religador ou fusível no início de trechos extensos, onde o nível mínimo de curto-circuito seja insuficiente para sensibilizar o dispositivo de proteção de retaguarda.

- Alocação de religador ou fusível, logo após cargas de grande importância e cuja continuidade de serviço deva ser elevada, caso o circuito após estas cargas seja extenso.

- Alocação de religador no início de ramais que alimentam cargas classificadas como especiais ou de grande importância.

- Alocação de religador no início de ramais que suprem cargas importantes localizadas em áreas sujeitas à alta incidência de faltas temporárias.

Para alocação de chaves seccionadoras os pontos candidatos são definidos considerando-se: 


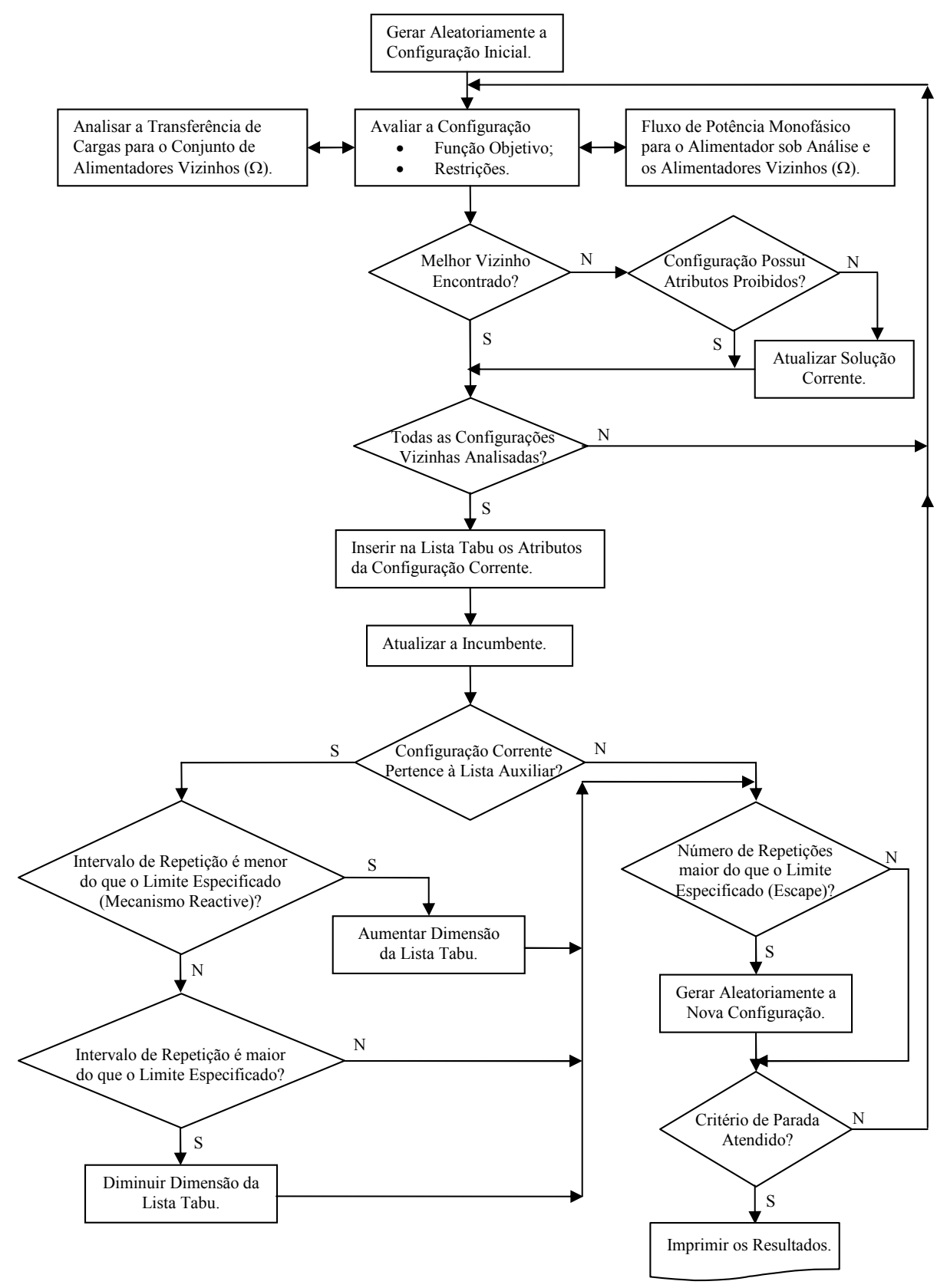

Figura 3: Diagrama de blocos do algoritmo BTR implementado. 
- Total de carga ou de consumidores que fazem parte de um determinado trecho da rede - seção;

- Natureza e importância da carga;

- Características sócio econômicas, de cada classe de consumidores - residenciais, comerciais, industriais, serviços públicos, escolas e hospitais.

- Índices de faltas permanentes e temporárias do conjunto de consumidores, de cada seção da rede de distribuição.

\subsection{Busca Tabu Reativa}

O modelo matemático para a alocação e/ou realocação de dispositivos de controle e proteção é um problema PNLB, e um algoritmo especializado BTR (Battiti, 1994) é proposto para resolvê-lo. Na Figura 3 ilustra-se o diagrama de blocos detalhado do algoritmo BTR implementado.

\subsubsection{Codificação}

Para mapear as possíveis soluções para o problema de alocação otimizada de dispositivos de controle e proteção é utilizada uma codificação em base decimal inteira conforme ilustrada na Figura 4.

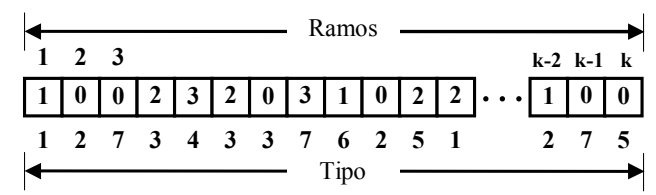

Figura 4: Esquema de Codificação Implementado - BTR.

Cada seção (ou ramo) do alimentador candidato é representada por um valor dependendo de qual dispositivo é alocado: 0 para nenhum dispositivo, 1 para religadores, 2 para chaves fusíveis, e 3 para chaves seccionadoras. O alimentador é dividido em seção principal e ramais laterais. É possível instalar qualquer tipo de dispositivo na seção principal. Os ramais laterais são subdivididos em duas categorias: $1^{\circ}$ Categoria lateral, onde todos os tipos de dispositivos de proteção podem ser instalados; e, $2^{\circ}$ Categoria lateral, onde só podem ser instaladas chaves fusíveis.

Cada região geográfica do alimentador definida anteriormente define um determinado tipo de seção em função dos dispositivos que são permitidos alocar. Sete tipos de seções são definidas, e os dispositivos permitidos para alocação, em cada caso, são os seguintes: Tipo 1: Religadores, Fusíveis, e Chaves Seccionadoras; Tipo 2: Religadores e Chaves Seccionadoras; Tipo 3: Religadores e Fusíveis; Tipo 4: Fusíveis e Chaves Seccionadoras;
Tipo 5: Fusíveis; Tipo 6: Religadores; Tipo 7: Chaves Seccionadoras.

\subsubsection{Configuração Inicial}

A BTR começa a partir de uma configuração inicial em que certo número de dispositivos de cada tipo está alocado no alimentador. Para gerar esta configuração efetua-se uma exploração no espaço de busca do alimentador sob análise para determinar uma quantidade adequada de dispositivos de controle e proteção. A quantidade máxima de dispositivos para a alocação e realocação é estabelecida através de critérios técnicos e econômicos.

\subsubsection{Estrutura de Vizinhança.}

Para o algoritmo BTR buscar novas soluções factíveis uma estrutura de vizinhança precisa ser definida. O algoritmo de BTR irá procurar uma solução melhor do que a solução corrente, em um conjunto de soluções vizinhas. Neste trabalho, duas estruturas de vizinhanças são definidas.

A primeira considera que a transição de uma configuração para outra é realizada para cada alimentador, segundo o esquema de codificação mantendo fixo o número de equipamentos, da seguinte forma:

a. O primeiro equipamento alocado na configuração corrente irá mudar de posição, os demais equipamentos continuam fixos;

b. A transição destes equipamentos é realizada para a primeira posição da configuração corrente que não possue equipamento alocado, obedecendo à restrição que contempla o tipo de seção e de dispositivo, o que define o primeiro vizinho de $N(x)$. A próxima transição é realizada para a segunda posição da configuração corrente que permite alocar este tipo de dispositivo, e assim sucessivamente, fazendo com que os equipamentos um a um, mudem para todas as posições da configuração corrente onde possam ser alocados. Ao final destas transições é obtido o conjunto de configurações vizinhas da configuração corrente $N(x)$.

A segunda estrutura de vizinhança refere-se à quantidade de cada tipo de dispositivo de controle e proteção que pode ser alocado com vistas a obter soluções técnicas e economicamente viáveis. Uma configuração vizinha da melhor configuração encontrada considerando-se um número fixo de dispositivos de controle e proteção é obtida aumentando uma unidade de cada vez, de cada um dos tipos de dispositivos. Desta forma esta estrutura de 
vizinhança consiste nos seguintes passos, considerando a melhor configuração vizinha encontrada:

i. Se o número de religadores que é permitido alocar não for superior ao máximo permitido, alocar aleatoriamente mais um religador no sistema, e realizar novamente a análise do primeiro tipo de vizinhança. Verificar se ocorreu melhoria da solução incumbente com o novo número de religadores alocados; e ir ao passo iv;

ii. Se o número de chaves seccionadoras que é permitido alocar não for superior ao máximo permitido, e também de outras análises deste tipo de vizinhança ocorreu melhoria da solução incumbente, alocar aleatoriamente uma chave seccionadora no sistema, e realizar novamente a análise do primeiro tipo de vizinhança. Verificar se ocorreu melhoria da solução incumbente com o novo número de chaves seccionadoras alocadas; e ir ao passo v;

iii. Se o número de fusíveis que é permitido alocar não for superior ao máximo permitido, e também de outras análises deste tipo de vizinhança ocorreu melhoria da solução incumbente, alocar aleatoriamente um elo fusível no sistema, e realizar novamente a análise do primeiro tipo de vizinhança. Verificar se ocorreu melhoria da solução incumbente com o novo número de chaves fusíveis alocadas; e ir ao passo vi;

iv. Se ocorrerem melhorias da solução incumbente aumentando o número de religadores voltar ao passo i. Caso contrário ir ao passo ii;

v. Se ocorrerem melhorias da solução incumbente aumentando o número de chaves seccionadoras, voltar ao passo ii. Caso contrário ir ao passo iii;

vi. Se ocorrerem melhorias da solução incumbente aumentando o número de fusíveis, voltar ao passo iii. Caso contrário parar a busca.

\section{TESTES E RESULTADOS}

A metodologia proposta foi implementada e testada em um alimentador real, 13,8 kV, 134-barras (LaPSEE, 2009). Este alimentador tem 8 linhas, interligações com 7 alimentadores vizinhos (duas interligações pertencem ao alimentador vizinho 3), como mostrado na Figura 5. Considerando os critérios apresentados na seção 3.1, trinta e seis (36) possíveis localizações foram selecionadas para a alocação de chaves seccionadoras, trinta e oito (38) para a alocação de fusíveis e onze (11) para a alocação de religadores. Taxas de faltas permanentes e temporárias (faltas/km/ano) utilizadas nos testes são, respectivamente 0,072 e 0,98 . Os parâmetros utilizados no algoritmo de BTR em todas as simulações são: tamanho da lista tabu inicial (LT) 10, tamanho lista tabu auxiliar 20, número de iterações 70 .

As informações da porcentagem dos tipos de consumidores residenciais, comerciais e industriais conectados aos alimentadores do sistema estão disponíveis no banco de dados da concessionária. No alimentador sob estudo são considerados que 50\% são consumidores residenciais, $30 \%$ consumidores comerciais e $20 \%$ consumidores industriais.

$\mathrm{Na}$ Tabela 1 encontram-se os custos de reparos e chaveamentos utilizados nos testes, e que foram adaptados de (Chowdhury and Koval, 1998). Os efeitos dos dispositivos de proteção, devido a sequência de religamentos, são considerados no modelo através do termo chamado CIT. Valores considerados para CTR, CTC e CTI são iguais a US\$ 1,00 .

Na Tabela 2 mostram-se os custos fixos dos dispositivos de controle e proteção. Estes custos compreendem os custos de aquisição do equipamento, instalação do material e os custos trabalhistas praticados no mercado Brasileiro. Custos fixos correspondentes aos religadores, fusíveis e chaves seccionadoras são apresentados na Tabela 3 . O horizonte de planejamento é de cinco anos, e a taxa de atualização anual dos custos de interrupção (I) é de $5 \%$ ao ano. As restrições consideradas para o alimentador de 134-barras são as seguintes:

- O número máximo de religadores que é permitido alocar é quatro (4);

- Não há limitação do número de chaves fusíveis para ser alocado;

- Fusíveis não podem ser instalados na seção principal do alimentador, nem em conexões com correntes de cargas futuras acima de $50 \mathrm{~A}$;

- Há um disjuntor com relé de religamento entre as barras $1-2$;

- Número máximo de fusíveis em série é igual a três (3);

- Fusíveis não podem ser alocados a montante dos religadores;

- A capacidade de fluxo dos alimentadores e a capacidade reserva das subestações não devem ser violadas.

Para validar a eficiência do modelo e da técnica de solução proposta, três casos com algumas condições de testes foram realizados com este alimentador real, ou seja:

1. Cinco diferentes condições de projetos são consideradas com diferentes números de religadores: $0,1,2$, 


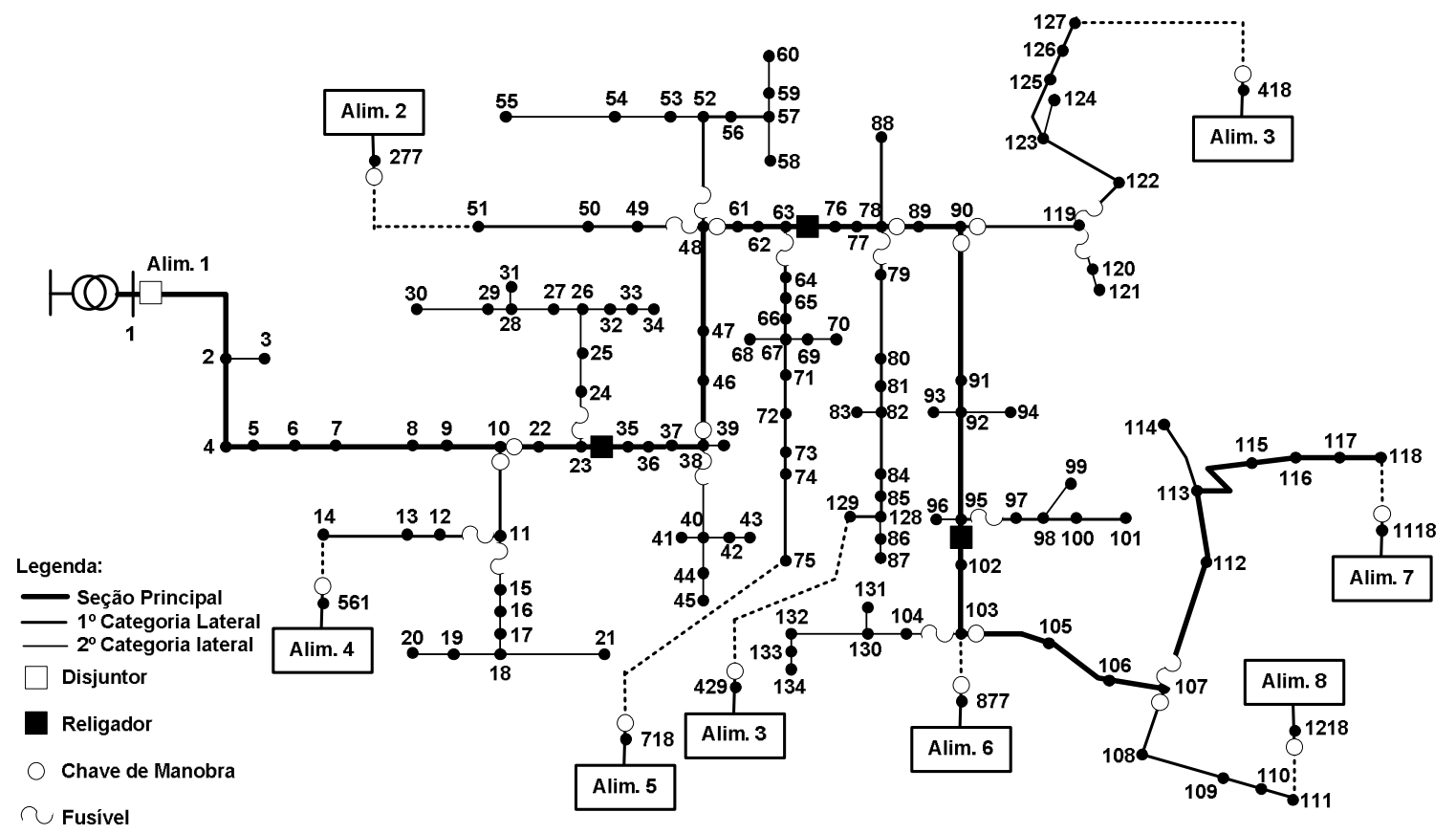

Figura 5: Alimentador de distribuição real, 13.8 kV, 134-barras, aéreo e trifásico.

Tabela 1: Custo de Reparo (CR) e Chaveamento (CC), por Categoria de Consumidores.

\begin{tabular}{|l|r|r|}
\hline Categoria & $\begin{array}{c}\text { CR (4 horas) } \\
\text { (US } \$ / \mathrm{kW})\end{array}$ & $\begin{array}{c}\text { CC (1,5 horas) } \\
\text { (US } \$ / \mathrm{kW})\end{array}$ \\
\hline Residencial & 6,00 & 0,40 \\
\hline Comercial & 120,00 & 60,00 \\
\hline Industrial & 18,54 & 9,62 \\
\hline
\end{tabular}

Tabela 2: Custos Fixos de Equipamento de Controle e Proteção.

\begin{tabular}{|l|r|}
\hline Equipamentos & Custo em US\$ \\
\hline Religador & $25.000,00$ \\
\hline Fusível & $1.000,00$ \\
\hline Chave de Manobra & $2.730,00$ \\
\hline
\end{tabular}

3 e 4. As melhores soluções encontradas para a alocação de religadores, chaves seccionadoras e fusíveis para cada teste são apresentadas na Tabela 3. Os custos correspondentes de interrupção temporária (CIT), custos de interrupção permanente (CIP), custos fixos $(\mathrm{CF})$ e o custo total de interrupção (CI) também são apresentados na Tabela 3. Na Figura 6 mostra como ocorre a variação de CIT, CIP, CF e CI em cada teste, devido à variação do número de religadores.
2. Os dispositivos de controle e proteção são alocados separadamente. $\mathrm{Na}$ primeira etapa as chaves seccionadoras são alocadas; e na segunda etapa, os dispositivos de proteção são alocados no alimentador. As mesmas condições do caso 2, são consideradas para realizar este teste. Na Tabela 4 apresentam-se os resultados para alocação independente de dispositivos de controle e proteção.

3. O teste é focado sobre o sistema de proteção existente, ou seja, avaliar qual é o nível de qualidade do sistema que está em funcionamento. Inicialmente, o índice CI é calculado com base nos dispositivos de controle e proteção existentes. Em seguida utilizando a metodologia proposta, simula-se a realocação otimizada dos dispositivos de controle e proteção. Na Tabela 5 apresentam-se os custos interrupção do sistema existente e os custos de interrupção do sistema após a realocação de dispositivos de controle e proteção.

\subsection{Análise dos Resultados Obtidos}

Na Figura 6 ilustra-se a validade do modelo matemático proposto. As soluções obtidas levam em conta a relação adequada entre custos de investimento $\times$ benefícios. O CIT apresenta um decaimento significativo até uma determinada quantidade de religadores instalados. Este comportamento do CIT depende das características das cargas e dos índices de faltas do alimentador. Para o alimentador sob análise, a 
alocação de mais que 2 religadores conduz a um cenário em que os termos da função objetivo não podem ser reduzidos pela alocação de mais religadores.

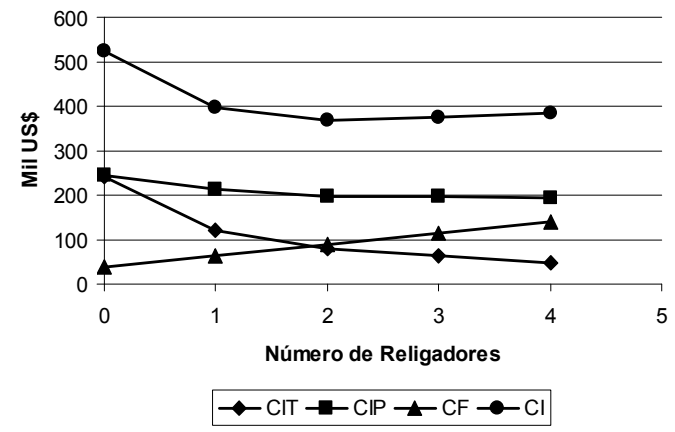

Figura 6: Termos da Função Objetivo $\times$ Número de Religadores.

Os resultados obtidos através do teste 1 da Tabela 3, mostram a importância que os religadores desempenham na confiabilidade do sistema. Nesta configuração, o disjuntor com relé de religamento, alocado entre a barra 1 e barra 2, é o único dispositivo com capacidade para eliminar as faltas temporárias. A sensibilidade deste dispositivo é limitada na sua zona de proteção. Faltas temporárias fora da sua zona de proteção são tratadas pela atuação de fusíveis, desconectando desnecessariamente o conjunto de consumidores no ponto em que a falta ocorreu. Portanto, um processo de restauração da rede é requerido. No modelo proposto, este aspecto é considerado no CIT, que apresenta um alto valor, em comparação com as outras configurações apresentadas na Tabela 3.

Na Tabela 4 apresentam-se os resultados para a alocação independente de dispositivos de controle e proteção, isto é, primeiro são alocadas as chaves seccionadoras, e depois são alocados os dispositivos de proteção. Os resultados obtidos para esta condição de teste são comparados com os resultados obtidos através da metodologia proposta, que considera simultaneamente alocação de dispositivos de controle e proteção no modelo matemático. Para a metodologia proposta os custos de interrupção obtidos são menores para a mesma quantidade de dispositivos alocados no alimentador. Essa característica do modelo proposto era esperada, uma vez que a inclusão da alocação de chaves seccionadoras junto com dispositivos de proteção aumenta as possibilidades de encontrar soluções otimizadas e de boa qualidade para o problema de confiabilidade da rede, uma vez que a técnica de solução proposta por meio de BTR não apresenta limitações computacionais para considerar modelos mais complexos. Na Tabela 5 apresenta-se a redução do custo de interrupção (CI) no sistema devido à realocação dos dispositivos de controle e proteção. A redução dos custos foi significativa, mesmo considerando os
Tabela 3: Melhores Soluções Encontradas.

\begin{tabular}{|c|c|c|c|}
\hline Testes & Dispositivos & $\begin{array}{l}\text { Localização do Dispositivo } \\
\text { Barra Inicial - Barra Final }\end{array}$ & Custos (US\$) \\
\hline \multirow{3}{*}{1} & Religadores & ---- & \multirow{3}{*}{$\begin{aligned} \mathbf{C I T} & =242,436.84 \\
\mathbf{C I P} & =243,376.68 \\
\mathbf{C F} & =39,570.00 \\
\mathbf{C I} & =525,383.52\end{aligned}$} \\
\hline & Fusíveis & $\begin{array}{l}10-11,23-24,38-40,48-49,48- \\
52,63-64,82-84,78-88,92-94, \\
95-97,103-104,107-108,107- \\
112,119-120,119-122 .\end{array}$ & \\
\hline & Chaves Seccionadoras & $\begin{array}{l}10-22,23-35,38-46,48-61,78- \\
79,78-89,90-91,103-105,90- \\
119 .\end{array}$ & \\
\hline \multirow{3}{*}{2} & Religadores & 63-76. & \multirow{3}{*}{$\begin{aligned} \mathbf{C I T} & =120,951.42 \\
\mathbf{C I P} & =212,264.11 \\
\mathbf{C F} & =64,570.00 \\
\mathbf{C I} & =397,785.53\end{aligned}$} \\
\hline & Fusíveis & $\begin{array}{l}10-11,23-24,38-40,48-49,52- \\
53,52-56,63-64,82-84,92-94, \\
95-97,103-104,107-108,107- \\
112,119-120,119-122 .\end{array}$ & \\
\hline & Chaves Seccionadoras & $\begin{array}{l}10-22,23-35,38-46,48-52,78- \\
79,78-89,90-91,103-105,90- \\
119 .\end{array}$ & \\
\hline \multirow{3}{*}{3} & Religadores & $38-46,90-91$. & \multirow{3}{*}{$\begin{aligned} \mathbf{C I T} & =80,124.19 \\
\mathbf{C I P} & =198,011.08 \\
\mathbf{C F} & =89,570.00 \\
\mathbf{C I} & =367,705.28\end{aligned}$} \\
\hline & Fusíveis & $\begin{array}{l}10-11,11-12,23-24,38-40,48- \\
49,48-52,63-64,78-79,78-88, \\
92-94,95-97,103-104,107-112, \\
119-120,119-122 .\end{array}$ & \\
\hline & Chaves Seccionadoras & $\begin{array}{l}10-22,23-35,48-61,63-76,78- \\
89,95-102,103-105,107-108, \\
90-119 .\end{array}$ & \\
\hline \multirow{3}{*}{4} & Religadores & $23-35,48-61,78-89$ & \multirow{3}{*}{$\begin{aligned} \mathbf{C I T} & =63,285.26 \\
\mathbf{C I P} & =195,419.41 \\
\mathbf{C F} & =114,570.00 \\
\mathbf{C I} & =373,274.67\end{aligned}$} \\
\hline & Fusíveis & $\begin{array}{l}10-11,11-12,23-24,38-40,48- \\
49,48-52,67-71,78-79,78-88, \\
98-100,103-104,107-108,107- \\
112,119-120,119-122 .\end{array}$ & \\
\hline & Chaves Seccionadoras & $\begin{array}{l}10-22,38-46,63-64,63-76,90- \\
91,95-97,95-102,103-105,90- \\
119 .\end{array}$ & \\
\hline \multirow{3}{*}{5} & Religadores & $23-35,48-61,78-89,103-105$. & \multirow{3}{*}{$\begin{aligned} \mathbf{C I T} & =49,025.17 \\
\mathbf{C I P} & =194,240.24 \\
\mathbf{C F} & =139,570.00 \\
\mathbf{C I} & =382,835.41\end{aligned}$} \\
\hline & Fusíveis & $\begin{array}{l}11-12,11-15,23-24,38-40,48- \\
49,48-52,63-64,82-84,78-88, \\
92-94,95-97,103-104,107-112, \\
119-120,119-122 .\end{array}$ & \\
\hline & Chaves Seccionadoras & $\begin{array}{l}10-11,10-22,38-46,63-76,78- \\
79,90-91,95-102,107-108,90- \\
119 .\end{array}$ & \\
\hline
\end{tabular}

Tabela 4: Resultado da alocação independente, primeiramente chaves de manobra em seguida alocação de dispositivos de proteção.

\begin{tabular}{|c|c|c|c|}
\hline Testes & Dispositivos & $\begin{array}{l}\text { Localização do Dispositivo } \\
\text { Barra Inicial - Barra Final }\end{array}$ & Custos (US\$) \\
\hline \multirow{3}{*}{1} & Religadores & $38-46,90-91$ & \multirow{3}{*}{$\begin{aligned} \mathbf{C I T} & =80,124.19 \\
\mathbf{C I P} & =227,340.25 \\
\mathbf{C F} & =89,570.00 \\
\mathbf{C I} & =397,034.45\end{aligned}$} \\
\hline & Fusíveis & $\begin{array}{l}11-12,11-15,26-27,26-32,40- \\
44,48-49,52-53,52-56,67-71, \\
82-84,95-97,103-104,107-112, \\
119-120,119-122 .\end{array}$ & \\
\hline & $\begin{array}{l}\text { Chaves } \\
\text { Seccionadoras }\end{array}$ & $\begin{array}{l}10-11,23-24,23-35,38-40,48- \\
52,63-64,78-79,78-89,103- \\
105 .\end{array}$ & \\
\hline
\end{tabular}


custos fixos (CF) do sistema existente, sendo igual a zero. O custo fixo da realocação está relacionado apenas com os custos de mão-de-obra necessários para realizar a realocação dos dispositivos de controle e proteção no alimentador.

\section{CONCLUSÕES}

Neste artigo apresenta-se uma nova abordagem de alocação e realocação integrada de dispositivos de controle e proteção em alimentadores de distribuição. O problema foi modelado como PNLB. Neste modelo consideram-se os principais aspectos físicos existentes no problema que afetam diretamente os custos de investimentos e de melhoria dos índices de confiabilidade das redes de distribuição.

A técnica proposta para encontrar soluções de boa qualidade cumpriu com as restrições físicas e operacionais impostas nos testes realizados em um alimentador real. Para cada teste é apresentado na Tabela 3 um conjunto de soluções de boa qualidade, incluindo a melhor solução que foi obtida. Considerando os procedimentos de operação e manutenção da concessionária, é possível que os engenheiros e técnicos possam analisar e decidir sobre a configuração mais adequada de acordo com a filosofia de confiabilidade utilizada pela empresa, dentro de um conjunto de soluções de boa qualidade fornecido pela metodologia.

A metodologia proposta permite uma análise da alocação de dispositivos de controle e proteção em projetos de novos sistemas, bem como a re-alocação para os sistemas de distribuição existentes.

Tabela 5: Resultados da comparação do sistema de controle e proteção existente, e com a realocação através da metodologia proposta.

\begin{tabular}{|c|c|c|c|}
\hline Testes & Dispositivos & $\begin{array}{l}\text { Localização do Dispositivo } \\
\text { Barra Inicial - Barra Final }\end{array}$ & Custos (US\$) \\
\hline \multirow{3}{*}{1 (Existente) } & Religadores & $9-10,90-91$ & \multirow{3}{*}{$\begin{aligned} \mathbf{C I T} & =109,643.08 \\
\mathbf{C I P} & =210,654.45 \\
\mathbf{C F} & =0,000.00 \\
\mathbf{C I} & =320,297.54\end{aligned}$} \\
\hline & Fusíveis & 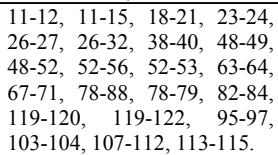 & \\
\hline & $\begin{array}{l}\text { Chaves } \\
\text { Seccionadoras }\end{array}$ & $\begin{array}{l}7-8,10-11,47-48,71-72,78- \\
89,102-103,107-108,112- \\
113,122-123 .\end{array}$ & \\
\hline \multirow{3}{*}{2 (Realocação) } & Religadores & $38-46,78-89$ & \multirow{3}{*}{$\begin{aligned} \mathbf{C I T} & =78,553.46 \\
\mathbf{C I P} & =206,124.43 \\
\mathbf{C F} & =14,968.00 \\
\mathbf{C I} & =299,645.89\end{aligned}$} \\
\hline & Fusíveis & $\begin{array}{l}11-15,18-19,18-21,23-24, \\
26-27,26-32,38-40,48-52, \\
52-53,52-56,63-64,67-71, \\
78-79,82-84,78-88,92-94, \\
95-97, \quad 103-104, \quad 107-108, \\
107-112,119-120,119-122 .\end{array}$ & \\
\hline & $\begin{array}{l}\text { Chaves } \\
\text { Seccionadoras }\end{array}$ & $\begin{array}{l}10-11,11-12,10-22,23-35, \\
48-49,48-61,90-91,103-105, \\
90-119 .\end{array}$ & \\
\hline
\end{tabular}

\section{AGRADECIMENTOS}

Os autores agradecem ao MCT, Fundo Setorial de Energia (CT-Energ) por intermédio do CNPq (Proc. 554661/2006-3), CNPq Proc. 301060/2006-1 e Fapesp (processo n o 02/04811-9) pelo financiamento desse projeto.

\section{REFERÊNCIAS}

Anderson, P. (1999). Power system protection, McGraw Hill and IEEE Press.

Baran, M. and Wu, F. (1989). Network reconfiguration in distribution systems for loss reduction and load balancing, IEEE Trans. on Power Delivery 4(2): 1401-1407.

Battiti, R. (1994). The reactive tabu search, ORSA J. Computing 6(2): 126-140.

Billinton, R. and Jonnavithula, S. (1996). Optimal switching device placement in radial distribution systems, IEEE Trans. on Power Systems 11(3): 1646-1651.

Brown, R., Gupta, S., Christie, R. and Venkata, S. (1996). A genetic algorithm for reliable distribution system design, Proceedings ISAP'96 International Conference on Intelligent System Applications to Power System 28(2): 29-33.

Celli, G. and Pilo, F. (1999). Optimal sectionalizing switches allocation in distribution networks, IEEE Trans. on Power Systems 14(3): 1167-1172.

Chowdhury, A. and Koval, D. (1998). Value-based distribution system reliability planning, IEEE Trans. on Industry Applications 34(1): 23-29.

LaPSEE (2009). Practical 135 bus feeder data, Available: http://www. dee.feis.unesp.br/lapsee/ TestSystems/135_Bus_Feeder.pdf.

Laboratório de Planejamento de Sistemas de Energia Elétrica (LaPSEE).

Levitin, G., Mazal-Tov, S. and Elmakis, D. (1994). Optimal sectionalizer allocation in electric distribution systems by genetic algorithm, Electric Power Systems Research 31: $97-102$.

Silva, L., Pereira, R., and Mantovani, J. R. S. (2004). Allocation of protective devices in distribution circuits using nonlinear programming models and genetic algorithms, Electric Power Systems Research 69(1): 77-84.

Soudi, F. and Tomsovic, K. (1999). Optimal distribution protection design: Quality of solution and 
computational analysis, International Journal on Electric Power and Energy Systems 21: 327-335.

Soudi, F. and Tomsovic, K. (2001). Optimal trade-offs in distribution protection design, IEEE Trans. on Power Delivery pp. 292-296.

Teng, J.-H. and Liu, Y.-H. (2003). A novel acs-based optimum switch relocation method, IEEE Trans. on Power Systems 18(1): 113-120. 